Dhaka Univ. J. Sci. 64(2): 109-113, 2016 (July)

\title{
Study on De Rham Cohomology Algebra of Manifolds
}

\author{
Saraban Tahora ${ }^{1^{*}}$ and Khondokar M. Ahmed ${ }^{2}$ \\ ${ }^{1}$ Department of Civil Engineering, University of Information Technology and Sciences (UITS), Dhaka-1212, Bangladesh \\ ${ }^{2}$ Department of Mathematics, Dhaka University, Dhaka-1000, Bangladesh
}

(Received: 3 September 2015; Accepted: 7 June 2016)

\begin{abstract}
In the present paper some aspects of exterior derivative, graded algebra, cohomology algebra, de Rham cohomology algebra, singular homology, cohomology class are studied. Graded subspace, smooth map, a singular $p$ - simplex in a manifold $M$, oriented $n$ - manifold $M$, the space of $p$ - cycles and $p$ - boundaries, $p^{\text {th }}$ singular homology and homology class are treated in our paper. A theorem 3.03 is established which is related to orientable manifold.
\end{abstract}

Keywords: De Rham cohomology, cohomology algebra, $p^{\text {th }}$ de Rham cohomology space, graded algebras, homology class and orientation class.

\section{Introduction}

The emergence of differential geometry as a distinct discipline is generally credited to Carl Friedrich Gauss and Bernhard Riemann. Riemann first described manifolds in his famous habilitation lecture before the faculty at Gottingen. He motivated the idea of a manifold by an intuitive process of varying a given object in a new direction. The concept of graded manifolds is developed by Manin. In the de Rham cohomology algebra of a smooth manifold is constructed by means of the calculus of differential forms which in turn is the natural global version of the usual differential calculus in $\mathbb{R}^{n}$. In the present paper, we have been discussed Cohomology Algebra, Singular Homology, Cohomology Class with some important theorems, lemmas, corollaries, propositions and examples.

\section{Cohomology Algebra}

Given an $n$ - manifold of $M$ and we consider the graded algebra [2]

$$
A(M)=\sum_{p=0}^{n} A^{p}(M)
$$

of differential forms on $M$.

It follows that the exterior derivatives makes $A(M)$ into a graded differential algebra. The co cycles in this differential algebra consist of the differential forms $\Phi$ which satisfy the condition $\delta \Phi=0$. Such a differential form is called closed. Since $\delta$ is an antiderivation, the closed forms are a graded subalgebra $Z(M)$ of $A(M)$.

Definition 1.01 Let $U \subseteq R^{n}$ be an open subset. Since $A^{0}(U)=C^{\infty}(U)$, we defined the exterior derivative by $d: A^{0}(U) \rightarrow A^{1}(U)$. For $p \geq 1$, we can define the exterior derivative

$$
d: A^{p}(U) \rightarrow A^{p+1}(U) .
$$

by the following formula:

$$
d\left(\sum_{1 \leq i_{1}<\cdots<i_{p} \leq n} f_{i_{1} \ldots . i_{p}} d x^{i_{1}} \wedge \ldots \ldots \wedge d x^{i_{p}}\right)
$$

\footnotetext{
* Author for correspondence. e-mail: sarabantahora@gmail.com
}

$$
=\sum_{1 \leq i_{1}<\cdots<i_{p} \leq n} d\left(f_{i_{1} \ldots i_{p}}\right) \wedge d x^{i_{1}} \wedge \ldots \ldots \wedge d x^{i_{p}} .
$$

It is clear that this operation is $\mathbb{R}$-linear.

Definition 1.02 The subset $B(M)=\delta A(M)$ is a graded ideal in $Z(M)$. The differential forms in $B(M)$ are called exact or coboundaries. The corresponding cohomology algebra is given by

$$
H(M)=Z(M) / B(M) .
$$

It is called the de Rham cohomology algebra [7] of $M$.

Lemma 1.03 The composition

$$
A^{p}(U) \stackrel{d}{\rightarrow} A^{p+1}(U) \stackrel{d}{\rightarrow} A^{p+2}(U)
$$

is trivial $\left(d^{2}=0\right)$.

Proof. The equation $d^{2}=0$ is equivalent to the equality of mixed partials which in turn, is equivalent to $\left[\frac{\partial}{\partial x^{k}}, \frac{\partial}{\partial x^{j}}\right]=0$, the commutatively of coordinate fields. By the antisymmetry of exterior multiplication, $d$ gives the same answer whether or not the indices are in increasing order or even are distinct. Thus

$$
\begin{aligned}
& d\left(d\left(f d x^{i_{1}} \wedge \ldots \ldots \wedge d x^{i_{p}}\right)\right) \\
= & d\left(\sum_{j=1}^{n} \frac{\partial f}{\partial x^{j}} d x^{j} \wedge d x^{i_{1}} \wedge \ldots \ldots \wedge d x^{i_{p}}\right) \\
= & \sum_{j=1}^{n} \sum_{k=1}^{n} \frac{\partial^{2} f}{\partial x^{k} \partial x^{j}} d x^{k} \wedge d x^{j} \wedge d x^{i_{1}} \wedge \ldots \ldots \ldots
\end{aligned}
$$

$\wedge d x^{i_{p}}$,

and this vanishes by the equality of mixed partials and the antisymmetry of exterior multiplication. Hence, the composition is trivial.

Corollary 1.04 If $U \subseteq \mathbb{R}^{n}$ and $V \subseteq \mathbb{R}^{m}$ are open subsets, and $\varphi: U \rightarrow V$ is smooth, then $d \circ \varphi^{*}=\varphi^{*} \circ d$ : $A^{p}(V) \rightarrow A^{p+1}(U)$, for all $p \geq 0$. 
Proof. Let $U \subseteq \mathbb{R}^{n}$ and $V \subseteq \mathbb{R}^{m}$ are open subsets and $\varphi: U \rightarrow V$ is open. Now,

$$
\begin{aligned}
& d\left(\varphi^{*}\left(f d y^{i_{1}} \wedge \ldots \ldots \wedge d y^{i_{p}}\right)\right) \\
& \quad=d\left(\varphi^{*}(f) \varphi^{*}\left(d y^{i_{1}}\right) \wedge \ldots \ldots \wedge \varphi^{*}\left(d y^{i_{p}}\right)\right) \\
& =d\left(\varphi^{*}(f) d\left(\varphi^{*}\left(y^{i_{1}}\right)\right) \wedge \ldots \ldots d\left(\varphi^{*}\left(y^{i_{p}}\right)\right)\right) \\
& =d\left(\varphi^{*}(f)\right) \wedge d\left(\varphi^{*}\left(y^{i_{1}}\right)\right) \wedge \ldots \ldots \wedge d\left(\varphi^{*}\left(y^{i_{p}}\right)\right) \\
& =\varphi^{*}(d f) \wedge \varphi^{*}\left(d y^{i_{1}}\right) \wedge \ldots \ldots \wedge \varphi^{*}\left(d y^{i_{p}}\right) \\
& =\varphi^{*}\left(d f \wedge d y^{i_{1}} \wedge \ldots \ldots \wedge d y^{i_{p}}\right) \\
& =\varphi^{*}\left(d\left(f d y^{i_{1}} \wedge \ldots \ldots \wedge d y^{i_{p}}\right)\right) .
\end{aligned}
$$

Since every $\eta \in A^{p}(V)$ is a sum of forms of the type used in the above computation, the claim follows.

Definition 1.05 For each integer $p \geq 0$, the $p$ th (de Rham) cohomology space of $M$ is the real vector space $H^{p}(M)=$ $Z^{p}(M) / B^{p}(M)$. If $\varphi: M \rightarrow N$ is smooth, the formula $\varphi^{*} \circ d=d \circ \varphi^{*}$ implies that

$$
\begin{aligned}
& \varphi^{*}\left(Z^{p}(N)\right) \subseteq Z^{p}(M) \\
& \varphi^{*}\left(B^{p}(N)\right) \subseteq B^{p}(M),
\end{aligned}
$$

so $\varphi^{*}$ induces an $\mathbb{R}$-linear map $\varphi^{*}: H^{p}(N) \rightarrow H^{p}(M)$.

Lemma 1.06 The graded $\mathbb{R}$-algebra $Z^{*}(M)$ is connected if and only if $M$ is a connected manifold [6].

Proof. The space $Z^{0}$ consists of all $f \in C^{\infty}(M)$ such that $d f=0$. That is, $Z^{0}(M)$ is the space of locally constant, real-valued functions on $M$. Identifying $\mathbb{R}$ with the space of constant functions in $C^{\infty}(M)$ we have $\mathbb{R} \subseteq Z^{0}(M)$. The product in $Z^{*}(M)$ of a constant function and a form becomes naturally identified with scalar multiplication. But locally constant functions are all constant of and only if $M$ is connected. This completes the proof.

Corollary 1.07 The space $H^{0}(M)$ is one-dimensional if and only if $M$ is connected. In this case, $H^{0}(M)=\mathbb{R}$ canonically. Generally, $H^{0}(M)$ is a direct product of copies of $\mathbb{R}$, one for each component of $M$.

Proof. The space $Z^{0}(M)$ consists of all $f \in C^{\infty}(M)$ such that $d f=0$. That is, $Z^{0}(M)$ is the space of locally constant, real valued functions on $M$. Identifying $\mathbb{R}$ with the space of constant functions in $C^{\infty}(M)$, we have $\mathbb{R} \subseteq Z^{0}(M)$. Indeed,

$$
H^{0}(M)=Z^{0}(M) / B^{0}(M)=Z^{0}(M),
$$

the space of locally constant functions and all the claims follow easily. Hence, the proof is complete.

Lemma 1.08 If $\operatorname{dim} M=n$, then $H^{p}(M)=0, \forall p>n$.

Proof. Let $\operatorname{dim} M=n$. We have to prove that $H^{p}(M)=$ $0, \forall p>n$. Indeed, $A^{p}(M)=0$ for all integers $p$ greater than the dimension of $M$. This means that $H^{p}(M)=$ $0, \forall p>n$ where $\operatorname{dim} M=n$. This completes the proof.
Definition 1.09 The graded algebra $H^{*}(M)$ is called the (de Rham) cohomology algebra of $M$. Whether or not it is connected, $H^{*}(M)$ has a unity, namely the constant function $1 \in Z^{0}(M)=H^{0}(M)$.

Theorem 1.10 The graded cohomology construction defines a contravariant $H^{*}$ from the category of differentiable manifolds and smooth maps to the category of anticommutative graded algebra over $\mathbb{R}$ and graded algebra homeomorphisms. The graded algebra $H^{*}(M)$ is connected iff $M$ is connected.

Lemma 1.11 The graded subspace $B^{*}(M) \subseteq Z^{*}(M)$ is a 2-sided ideal, hence $H^{*}(M)=Z^{*}(M) / B^{*}(M)$ is a graded, anticommutative algebra over the field $\mathbb{R}$.

Proof. If $w \in Z^{p}(M)$ and $\eta \in B^{q}(M), q \geq 1$, then $\eta=d \alpha$ for some $\alpha \in A^{p-1}(M)$, hence

$$
\begin{aligned}
w \wedge \eta=w & \wedge d \alpha \\
& =d w \wedge(-1)^{p} \alpha+(-1)^{p} w \wedge d\left((-1)^{p} \alpha\right) \\
= & d\left(w \wedge(-1)^{p} \alpha\right) .
\end{aligned}
$$

Since $\eta \wedge w=(-1)^{p q} w \wedge \eta$, it follows that $B^{*}(M)$ is a 2-sided ideal in $Z^{*}(M)$.

Definition 1.12 The (de Rham) cohomology algebra [1] with compact supports is $H_{c}^{*}(M)=Z_{c}^{*}(M) / B_{c}^{*}(M)$. Now, $H^{*}(M)=H_{c}^{*}(M)$ if and only if $M$ is compact.

Definition 1.13 A smooth map $\varphi: M \rightarrow N$ is proper, if for each compact set $C \subseteq N$, the set $\varphi^{-1}(C)$ is also compact.

Example $1.14 \mathrm{id}: M \rightarrow M$ is always proper. If $M$ is compact, $\varphi$ is always proper.

Definition 1.15 The space of compactly supported $p$-forms on $M$ is denoted by $A_{c}^{p}(M)$. Thus each $A_{c}^{p}(M)$ is a module over $C^{\infty}(M)$. The exterior product of two compactly supported forms is compactly supported.

Definition 1.16 If $\varphi: M \rightarrow N$ is proper and if $w \in$ $A_{c}^{p}(N)$, then

$$
\varphi^{*}(w) \in A_{c}^{p}(M) .
$$

As usual $\varphi^{*} \circ d=d \circ \varphi^{*}$, so the induced homomorphism of graded algebras [2] is

$$
\varphi^{*}: H_{c}^{*}(N) \rightarrow H_{c}^{*}(M) .
$$

\section{Singular Homology}

A singular $p$-simplex in a manifold $M$ is a smooth map $s: \Delta_{p} \rightarrow M$. Thus each point of $M$ can be thought of as a singular 0 -simplex and smooth curves, up to parametrization, are singular 1 -simplices. Now for $0 \leq i \leq$ $p$, the $i^{\text {th }}$ face of the singular $(p-1)$-simplex $F_{i}$ : $\Delta_{p-1} \rightarrow \Delta_{p}$ defined by

$$
\begin{aligned}
& F_{i}\left(x^{1}, \ldots \ldots, x^{p-1}\right) \\
& = \begin{cases}\left(x^{1}, \ldots \ldots, x^{i-1}, 0, x^{i}, \ldots \ldots, x^{p-1}\right) & \text { if } i>0 \\
\left(1-x^{1}-\cdots-x^{p-1}, x^{1}, \ldots \ldots, x^{p-1}\right) & \text { if } i=0 .\end{cases}
\end{aligned}
$$


The $0^{\text {th }}$ face of $\quad \Delta_{0}$ is empty. If $s: \Delta_{p} \rightarrow M$ is a singular $p$-simplex, the $i^{\text {th }}$ face of $s$ is the singular $(p-1)$-simplex $\partial_{i} s=s \circ F_{i}$.

Definition 2.01 The orientation of $M$ induces an orientation of $\partial M$. Let $\left\{U_{\alpha}, x_{\alpha}^{1}, \ldots \ldots, x_{\alpha}^{n}\right\}_{\alpha \in \mathfrak{A}}$ be an $\mathbb{H}^{n}$ atlas on $M$ respecting the orientation. Let $\mathfrak{U}^{\prime}=$ $\left\{\alpha \in \mathfrak{A} \mid U_{\alpha} \cap \partial M \neq \emptyset\right\}$ and consider the $\mathbb{R}^{n-1}$ - atlas

$$
\left\{U_{\alpha} \cap \partial M, x_{\alpha}^{2}, \ldots \ldots, x_{\alpha}^{n}\right\}_{\alpha \in \mathfrak{A}^{\prime}}
$$

of $\partial M$. This $\mathbb{R}^{n-1}$ - atlas on $\partial M$ defines an orientation of $\partial M$.

Theorem 2.02 For each oriented $n$-manifold $M$ [3], there is unique $\mathbb{R}$-linear function

$$
\int_{M}: A_{c}^{n}(M) \rightarrow \mathbb{R},
$$

called the integral and having the following property:

if $(U, \varphi)$ is an- orientation respecting coordinate chart, if $W \in A_{c}^{n}(M)$ has $\operatorname{supp}(w) \subset U$, and if

$$
\varphi^{-1 *}(w)=g d x^{1} \wedge \ldots \ldots \wedge d x^{n} \in A_{c}^{n}(\varphi(U)),
$$

then $\int_{M} w=\int_{\varphi(U)} g$ (The Riemann integral).

Proof. First we prove the uniqueness. Let $\left\{\left(U_{\alpha}, \varphi_{\alpha}\right)\right\}_{\alpha \in \mathfrak{A}}$ be smooth $\mathbb{H}^{n}$ - atlas on $M$ respecting the orientation. Let $\left\{\lambda_{\alpha}\right\}_{\alpha \in \mathfrak{A}}$ be a smooth partition of unity subordinate to the atlas. If $w \in A_{c}^{n}(M)$, then $\lambda_{\alpha} w \in A_{c}^{n}(M)$ and $\lambda_{\alpha} w \neq 0$ for only a finite number of $\alpha \in \mathfrak{U}$. This is because $\operatorname{supp}(w)$ is compact and the partition of unity is locally finite. Thus

$$
w=\sum_{\alpha \in \mathfrak{A}} \lambda_{\alpha} w
$$

and this sum is actually finite. Then, if $\int_{M}$ exists, linearity gives

$$
\int_{M} w=\sum_{\alpha \in \mathfrak{A}} \int_{M} \lambda_{\alpha} w
$$

and $\operatorname{supp}\left(\lambda_{\alpha} w\right)=\operatorname{supp}\left(\lambda_{\alpha}\right) \cap \operatorname{supp}(w)$ is a compact subset of $U_{\alpha}$. By the local property of $\int_{M}$, each $\int_{M} \lambda_{\alpha} w$ is uniquely given as

$$
\int_{M} \lambda_{\alpha} w=\int_{\varphi_{\alpha}\left(U_{\alpha}\right)}\left(\lambda_{\alpha} \circ \varphi_{\alpha}{ }^{-1}\right) g_{\alpha}
$$

where $g_{\alpha} d x^{1} \wedge \ldots \ldots \wedge d x^{n}=\varphi_{\alpha}{ }^{-1 *}\left(w \mid U_{\alpha}\right)$.

If $w \in A_{c}^{n}(M)$, only finitely many $\lambda_{\alpha} w$ are not identically 0 . Define

$$
\int_{M} \lambda_{\alpha} w=\int_{\varphi_{\alpha}\left(U_{\alpha}\right)}\left(\lambda_{\alpha} \circ \varphi_{\alpha}{ }^{-1}\right) g_{\alpha},
$$

where $g_{\alpha} d x^{1} \wedge \ldots \ldots \wedge d x^{n}=\varphi_{\alpha}{ }^{-1 *}\left(w \mid U_{\alpha}\right)$. Then we define a finite sum

$$
\int_{M} w=\sum_{\alpha \in \mathfrak{A}} \int_{M} \lambda_{\alpha} w,
$$

where, $\int_{M}: A_{c}^{n}(M) \rightarrow \mathbb{R}$ is a $\mathbb{R}$ linear map.

We must check that, if $\operatorname{supp}(w) \subset U$, where $(U, \varphi)$ is an arbitrary orientation respecting coordinate chart and if

$$
\varphi^{-1 *}(w)=g d x^{1} \wedge \ldots \ldots \wedge d x^{n},
$$

then $\int_{M} w=\int_{\varphi(U)} g$.

First we remark that

$$
\begin{gathered}
\int_{M} w=\sum_{\alpha \in \mathfrak{A}} \int_{\varphi_{\alpha}\left(U_{\alpha}\right)}\left(\lambda_{\alpha} \circ \varphi_{\alpha}^{-1}\right) g_{\alpha} \\
=\sum_{\alpha \in \mathfrak{A}} \int_{\varphi_{\alpha}\left(U_{\alpha} \cap U\right)}\left(\lambda_{\alpha} \circ \varphi_{\alpha}^{-1}\right) g_{\alpha},
\end{gathered}
$$

since $\operatorname{supp}(w) \subset U$. Now,

$$
\begin{aligned}
& \int_{\varphi_{\alpha}\left(U_{\alpha} U\right)}\left(\lambda_{\alpha} \circ \varphi_{\alpha}{ }^{-1}\right) g_{\alpha} \\
& =\int_{\varphi\left(U_{\alpha} \cap U\right)}\left(\lambda_{\alpha} \circ \varphi^{-1}\right) g,
\end{aligned}
$$

for each $\alpha \in \mathfrak{A}$. The fact that the charts are compatibly oriented is essential. Thus,

$$
\begin{aligned}
\int_{M} w & =\sum_{\alpha \in \mathfrak{A}} \int_{\varphi\left(U_{\alpha} \cap U\right)}\left(\lambda_{\alpha} \circ \varphi^{-1}\right) g \\
& =\sum_{\alpha \in \mathfrak{A}} \int_{\varphi(U)}\left(\lambda_{\alpha} \circ \varphi^{-1}\right) g \\
& =\int_{\varphi(U)}\left\{\sum_{\alpha \in \mathfrak{A}} \lambda_{\alpha} \circ \varphi^{-1}\right\} g \\
= & \int_{\varphi(U)} g . \\
\therefore \int_{M} w & =\int_{\varphi(U)} g .
\end{aligned}
$$

Theorem 2.03 Let $M$ be an oriented $n$-manifold and let $i: \partial M \hookrightarrow M$ be the inclusion. Then if $w \in A_{c}^{n-1}(M)$,

$$
\int_{M} d w=\int_{\partial M} i^{*}(w),
$$

where if $\partial M=\varnothing$, the right hand side is interpreted as 0 .

Theorem 2.04 Let $M$ be an oriented $n$-manifold with $\partial M=\emptyset$. Then $\int_{M}: H_{c}^{n}(M) \rightarrow \mathbb{R}$ is a well defined $\mathbb{R}$ linear surjection.

Proof. We know that $A_{c}^{n+1}(M)=0$, then we have,

$$
Z_{c}^{n}(M)=A_{c}^{n}(M) \text {. }
$$

If $w=d \eta \in B_{c}^{n}(M)$, then Stokes theorem and the fact that $\partial M=\emptyset$ imply that

$$
\int_{M} w=\int_{M} d \eta=\int_{\partial M} \eta=0 .
$$

Thus, the linear map

$$
\int_{M}: Z_{c}^{n}(M) \rightarrow \mathbb{R},
$$

induces a well defined linear map

$$
\int_{M}: H_{c}^{n}(M) \rightarrow \mathbb{R}
$$

To prove surjectivity, we only need prove that this map is nontrivial. Let $\left(U, x^{1}, \ldots \ldots, x^{n}\right)$ be a compatibly oriented 
chart and let $\lambda \in C^{\infty}(M)$ have compact support contained in $U$, with $\lambda \geq 0$ everywhere and $\lambda>0$ somewhere. Thus $w=\lambda d x^{1} \wedge \ldots \ldots \wedge d x^{n}$ can be interpreted as an element of $Z_{c}^{n}(M)$ and of $A_{c}^{n}\left(\mathbb{R}^{n}\right)$, so, $\int_{M} w=\int_{\mathbb{R}^{n}} \lambda>$ 0 .

This completes the required proof.

Definition 2.05 If $s: M_{p} \rightarrow M$ is a singular $p$-simplex and $\quad w \in A^{p}(M)$, then $s^{*}(w)$ has the form $g d x^{1} \wedge \ldots \ldots \wedge d x^{p}$ and we set

$$
\int_{s} w=\int_{\Delta_{p}} g
$$

where the right hand side is the Riemann integral [4]. If $s:\{0\} \rightarrow M$ is a singular 0 -simplex and $w=f \in A^{0}(M)$, the integral is interpreted to mean

$$
\int_{s} f=f(s(0)) .
$$

Definition 2.06 The standard $p$-simplex $\Delta_{p} \subset \mathbb{R}^{p}$ is the covex hull of the set $\left\{e_{0}, e_{1}, \ldots \ldots, e_{p}\right\}$, where $e_{i}$ is the $i^{\text {th }}$ standard basis vector, $1 \leq i \leq p$ and $e_{0}=0$.

Corollary 2.07 A form $w \in A^{p}(M)$ is closed if and only if $\int_{\partial s} w=0$, for every singular $(p+1)-\operatorname{simplex} s$ in $M$.

Proof. Let $w \in A^{p}(M)$. If $w$ is closed, then

$$
\int_{\partial s} w=\int_{S} d w=\int_{s} 0=0 .
$$

For the converse, suppose that $d w=\eta \neq 0$. Choose a point $x \in M$ such that $\eta_{x} \neq 0$. Choose vectors $v_{1}, \ldots \ldots, v_{p+1} \in$ $T_{x}(M)$ such that $\eta_{x}\left(v_{1} \wedge \ldots \ldots \wedge v_{p+1}\right)>0$. These vectors must be linearly independent, so we find a local coordinate chart $\left(U, x^{1}, \ldots \ldots, x^{n}\right)$ about $x$ in which $v_{i}$ is the value of ith coordinate field $\xi_{i}=\partial / \partial x^{i}$ at $x, 1 \leq i \leq p+1$. By making this chart is sufficiently small, we can guarantee that $\eta\left(\xi_{1} \wedge \ldots \ldots \wedge \xi_{p+1}\right)>0$ on all of $U$. Let $s: \Delta_{p} \rightarrow U$ be orientation-preserving, smooth imbedding into the coordinate $(p+1)$-plane $\left\{\left(x^{1}, \ldots \ldots, x^{n}\right) \in U \mid x^{p+2}=\right.$ $\ldots=x n=0$. It follows that

$$
\int_{\partial s} w=\int_{s} d w=\int_{s} \eta=0 .
$$

This completes the poof.

Definition 2.08 The space $Z_{p}(M) \subseteq C_{p}(M)$ of all $p$-cycles is the kernel of the boundary operator $\partial: C_{p}(M) \rightarrow$ $C_{p-1}(M)$. The space $B_{p}(M) \subseteq C_{p}(M)$ of all $p$ boundaries is the image of the boundary operator $\partial$ : $C_{p+1}(M) \rightarrow C_{p}(M)$.

Definition 2.09 The $p^{\text {th }}$ singular homology of $M$ is the vector space

$$
H_{p}(M)=\frac{Z_{p}(M)}{B_{p}(M)} .
$$

If $z \in Z_{p}(M)$, the homology class [5] of $z$ is the coset $[z] \in H_{p}(M)$ represented by the cycle $z$.

Theorem 2.10 If $M$ is a contractible $n$-manifold, then

$$
H_{p}(M)= \begin{cases}\mathbb{R}, & p=0 \\ 0, & p>0\end{cases}
$$

in particular, this is true for $M=\mathbb{R}^{n}$.

Proposition 2.11 If $w \in Z^{p}(M)$ and $z \in Z_{p}(M)$, then the real number $\int_{z} w$ depends only on the cohomology class $[w] \in H(M)$ and the homology class $[z] \in H_{p}(M)$.

Proof. Indeed, $[w]$ is the set of all closed $p$-forms $w+d \eta$, where $\eta \in A^{p-1}(M)$. We have

$$
\int_{z} d \eta=\int_{\partial z} \eta=\int_{0} \eta=0,
$$

by Stokes theorem and the fact that $z$ is a cycle, so

$$
\int_{z} w+d \eta=\int_{z} w
$$

Similarly, $[z]$ is the set of all $p$-cycles of the from $z+\partial c$, where $c \in C_{p+1}(M)$. Since $\int_{\partial c} w=\int_{c} \partial w=$ $\int_{c} 0=0$, we obtain,

$$
\int_{z+\partial c} w=\int_{z} w+\int_{\partial c} w=\int_{z} w
$$

This completes the proof.

\section{Cohomology Class}

Definition 3.01 The unique cohomology class $w_{M} \in H_{c}^{n}(M)$ which satisfies

$$
\int_{M}^{\#} w_{M}=1
$$

is called the orientation class for $M$.

Theorem 3.02 Let $M$ be a oriented $n$-manifold. Then

$$
\int_{M}^{\#}: H_{c}^{n}(M) \rightarrow \mathbb{R}
$$

is a linear isomorphism. Moreover,

$$
\operatorname{ker} \int_{M}=\partial\left(A_{c}^{n-1}(M)\right) \text {. }
$$

Theorem $3.03 H_{c}^{n}(\widetilde{M})=\left(H_{c}^{n}\right)_{-}(\widetilde{M}) ;\left(H_{c}^{n}\right)_{+}(\widetilde{M})=0$.

Proof. Since $\widetilde{M}$ is connected and orientable [6], $\operatorname{dim} H_{c}^{n}(\widetilde{M})=1$. Since

$$
H_{c}^{n}(\widetilde{M})=\left(H_{c}^{n}\right)_{+}(\widetilde{M}) \oplus\left(H_{c}^{n}\right)_{-}(\widetilde{M})
$$

it is sufficient to prove that $\left(H_{c}^{n}\right)_{-}(\widetilde{M}) \neq 0$.

Orient $\tilde{M}$ and let $\Omega \in A_{c}^{n}(\widetilde{M})$ be positive. Since $\tau$ reverses orientations,

$$
\Phi=\Omega=\tau^{*} \Omega
$$

is again positive. Hence $\int_{\widetilde{M}} \Phi>0$; i. e. $\Phi$ represents a nontrivial cohomology class

$\alpha \in H_{c}^{n}(\widetilde{M})$. But $\tau^{*} \Phi=-\Omega$; Thus

$\alpha \in\left(H_{c}^{n}\right)_{-}(\tilde{M})$ and so $H_{c_{-}}^{n}(\tilde{M}) \neq 0$.

Hence the Theorem is proved. 


\section{Conclusion}

We have focused some important preliminaries and fundamental definitions, examples and theorems which is essential to present this paper. By using de Rham cohomology algebra, grade cohomology construction, space of compactly supported $p$-forms on manifolds, singular $p$ - simplex of a manifold, oriented $n$-manifold the theorem 3.03 is established and so on.

\section{References}

1. Ahmed, K. M., 2002. A note on sheaves and cohomology on manifold, Ganit : Journal of the Bangladesh Mathematical Society, 22: 41-50.

2. Ahmed, K. M., 2007. A study of Graded manifolds, Dhaka Uni. J. Sci. 55(1): 35-39.

3. Ahmed, K. M., 2006. Study on Cohomology of Supermanifolds, BRAC University Journal, III, (1), 59-63.
4. Ahmed, K. M., Md. Showkat Ali, 2008. A study of spaces of constant curvature on Riemannian manifolds, Dhaka Uni. J. Sci. 56(1): 65-67.

5. Ahmed, K. M., Maruf Ahmed, Gulshan Khatun, Fardousi Ara Begum and M. Shahabuddin, Taposh K. Das and Satrajit Kumar Saha, 2011. Study on Cohomology of De Witt Suppermanifolds, of Jahangirnagar University Journal of Science ( ISSN 1022-8594 JUJS), 34: 89-96.

6. Ahmed, K. M., 2003. A note on affine connection on differentiable manifold, The Dhaka University Journal of Science, 52(1): 63-68.

7. Gereub, W. S. Haperin and Vanstone, 1972. R. De Rham Cohomology of Manifolds and Vector Bundles, 1. Academic press, New York. 
